"This is an Accepted Manuscript of an article published by Macromol. Symp., Wiley, vol 370, no 1, pgs 120-127, in Dec 2016."

\title{
Sensor Array for Volatile Organic Compounds based on Doped Poly (2,5-dimethyl aniline)
}

\author{
Katherine M. E. Stewart and Alexander Penlidis*
}

Institute for Polymer Research, Department of Chemical Engineering, University of Waterloo, 200 University Avenue West, Waterloo, Ontario, N2L3G1, Canada

Summary: A sensor array containing five different sensing materials has been created that is able to distinguish between six different volatile organic compounds (VOCs) at an analyte concentration of $5 \mathrm{ppm}$. While none of the sensing materials, poly (2,5-dimethyl aniline) (P25DMA) and P25DMA doped with four different metal oxide nanoparticles $\left(\mathrm{Al}_{2} \mathrm{O}_{3}, \mathrm{NiO}, \mathrm{TiO}_{2}\right.$, and $\left.\mathrm{ZnO}\right)$, were selective towards any single analyte on their own, through the use of a filtering algorithm, this partial selectivity was exploited. Principal component analysis (PCA) was used as that a filtering algorithm, which was able to separate the responses from the sensing materials and identify six different gas analytes (acetaldehyde, acetone, benzene, ethanol, formaldehyde, and methanol). Both unknown gas analytes and a gas mixture were evaluated to determine the effectiveness of this sensor array.

Keywords: sensor array; volatile organic compounds (VOCs); gas sensor; principal component analysis (PCA); metal oxide dopant

\section{Introduction}

Indoor air quality is important, especially now when many people spend the majority of their time indoors. ${ }^{[1]}$ Indoor air can be polluted with a variety of volatile organic compounds (VOCs). Two VOCs of great interest are formaldehyde and benzene. ${ }^{[2]}$ By monitoring levels of VOCs, specifically benzene and formaldehyde, it is possible to determine the quality of air inside a building.

To accomplish this goal of detecting multiple gas analytes simultaneously, a sensor array can be used. A sensor array combines multiple partially selective sensing materials, and 
using a filtering algorithm (such as principal component analysis (PCA)), is able to detect multiple gas analytes simultaneously. ${ }^{[3]}$ An unknown gas sample is compared to a reference or calibration plot and the unknown gas sample is identified based on where the response(s) from the sensor array fall on the plot in relation to the reference gas analytes. ${ }^{[4,5]}$

Polymeric sensing materials are ideal because they can be tailor-made to attract a specific gas and thus, can have high selectivity. ${ }^{[6]}$ This can be done by adding one or more side chains, a dopant (small amount of another material such as a metal oxide), or creating a copolymer. ${ }^{[7]}$ Polymeric sensors work mainly at low temperatures (below $100^{\circ} \mathrm{C}$ ) and are relatively inexpensive. ${ }^{[8]}$

Poly (2,5-dimethyl aniline) (P25DMA) and P25DMA doped with four different metal oxide nanoparticles are used for this sensor array. Both P25DMA and P25DMA doped with metal oxides have been used as sensing materials for different gas analytes. ${ }^{[9]}$ In addition, the four metal oxides used as dopants $\left(\mathrm{Al}_{2} \mathrm{O}_{3},{ }^{[10,11]} \mathrm{NiO},{ }^{[12,13]} \mathrm{TiO}_{2},{ }^{[14,15]}\right.$ and $\mathrm{ZnO}^{[16,17]}$ have been used as sensing materials for a variety of gas analytes.

These five sensing materials (P25DMA and P25DMA doped with $\left(\mathrm{Al}_{2} \mathrm{O}_{3}, \mathrm{NiO}\right.$, $\mathrm{TiO}_{2}$, and $\mathrm{ZnO}$ ) were evaluated as sensing materials individually first, then combined to produce a sensor array. PCA was used as the filtering algorithm and was able to distinguish between six different VOCs (acetaldehyde, acetone, benzene, ethanol, formaldehyde, and methanol).

\section{Experimental}

\subsection{Polymer Nanocomposite Synthesis}

The doped and undoped P25DMA was synthesized in the same manner, except no dopant was present when synthesizing the P25DMA. In a flask, $0.39 \mathrm{~mL}$ of 2,5-dimethyl aniline (A.C.S. reagent, Sigma-Aldrich, Oakville, Ontario, Canada), the equivalent of 5 wt. \% (based on the monomer weight) of the metal oxide dopant, and $20 \mathrm{~mL}$ of deionized water were sonicated (VWR B1500A-MT Ultrasonicator, Mississauga, Ontario, Canada) for 30 minutes. The four metal oxide nanoparticles used were $\mathrm{Al}_{2} \mathrm{O}_{3}$ (particle size $<50 \mathrm{~nm}$, $10 \mathrm{wt}$. $\%$ dispersion in water, Sigma-Aldrich, Oakville, Ontario), NiO (particle size $<50 \mathrm{~nm}$, concentration of 99.8\%, Sigma Aldrich, Oakville, Ontario, Canada), $\mathrm{TiO}_{2}$ (particle size 21 $\mathrm{nm}$, concentration of 99.5\%, Sigma-Aldrich, Oakville, Ontario, Canada), and ZnO (particle 
size $<100$ nm, 50 wt. \% in water, Sigma-Aldrich, Oakville, Ontario, Canada).

The solution was subsequently cooled down to $-1{ }^{\circ} \mathrm{C}$, then $1.0 \mathrm{~g}$ of ammonium persulfate dissolved in $5 \mathrm{~mL}$ of deionized water was added to the cooled solution. This mixture was swirled for 60 seconds, then left to polymerize for 6 hours. The resulting polymeric materials were filtered using a Büchner funnel and Whatman \#5 filter paper. The polymeric materials were washed using acetone then left to air dry.

In total five polymeric materials were synthesized: P25DMA, P25DMA doped with 5 wt. $\% \mathrm{Al}_{2} \mathrm{O}_{3}$ (P25DMA 5\% $\mathrm{Al}_{2} \mathrm{O}_{3}$ ), P25DMA doped with 5 wt. \% NiO (P25DMA 5\% NiO), P25DMA doped with 5 wt. \% $\mathrm{TiO}_{2}$ (P25DMA 5\% $\mathrm{TiO}_{2}$ ), and P25DMA doped with 5 wt. \% ZnO (P25DMA 5\% ZnO).

To measure the amount of gas analyte, $0.120 \mathrm{~g}$ of polymeric material was placed into 100 $\mathrm{mL}$ round bottom flask with $20 \mathrm{~mL}$ of ethanol. The polymer was slowly swirled around the bottom of the flask to distribute it, and then the ethanol was evaporated off in an oven at 60 ${ }^{\circ} \mathrm{C}$ for 18 hours. The polymeric materials were allowed to cool to room temperature before evaluation.

\subsection{Gas Test Set-up}

To evaluate the potential sensing materials, gas sorption tests were performed. Each sensing material was exposed to a known concentration of analyte (e.g. 5 ppm ethanol gas in a balance of nitrogen) and the amount of analyte that sorbed onto the sample was measured. Measurements were conducted at room temperature $\left(21^{\circ} \mathrm{C}\right)$ and slightly above atmospheric pressure (15 psi).

Gas analytes were mixed, if necessary, using an inline passive mixer, after which the gas line ran through an MKS RS-485 mass flow controller to regulate the flow at $200 \mathrm{sccm}$ and into the $100 \mathrm{~mL}$ round bottom flask containing the polymeric material. The gases then flowed out of the flask and through an MKS 640A pressure controller and MKS 1179A flow meter to ensure the pressure and flow rate were maintained at 15 psi and $200 \mathrm{sccm}$, respectively. Finally, the gas flowed into a specialized Varian 450 gas chromatograph (GC) with a photon discharge helium ionization detector (PDHID), capable of measuring down 
to the ppb level for different compounds (Figure 1). ${ }^{[18]}$

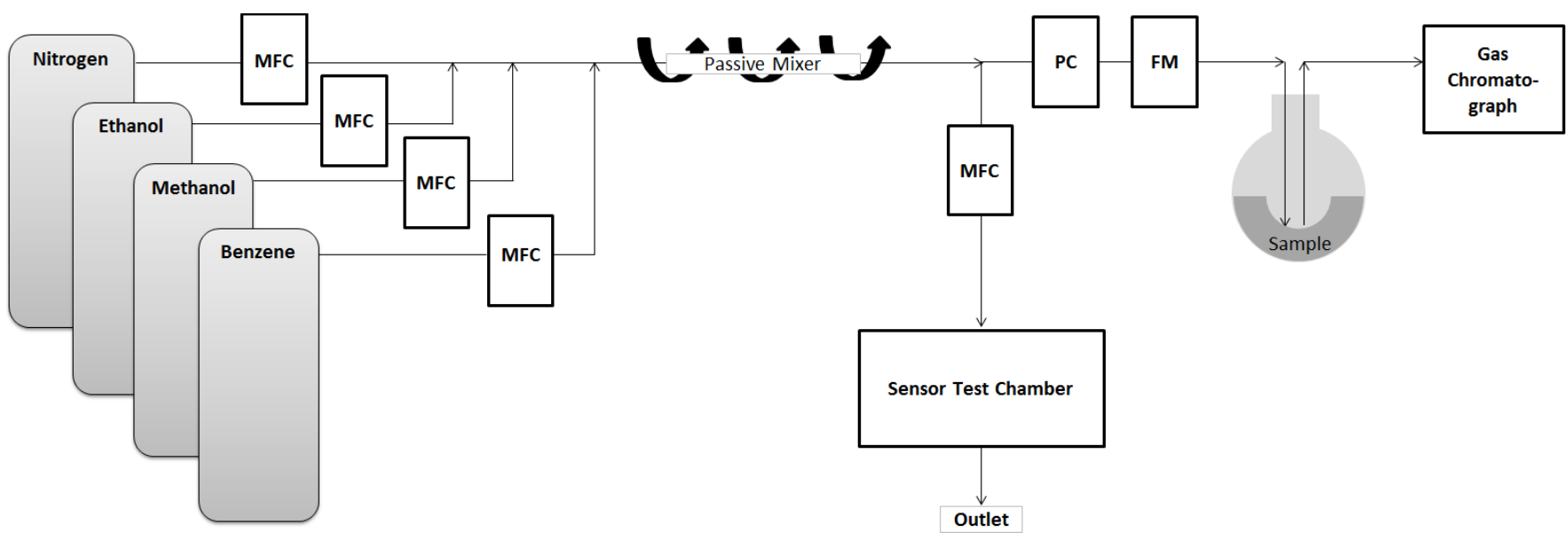

Figure 1. Schematic of the test system, where MFC, PC, and FM are mass flow controller, pressure controller, and flow meter, respectively.

The gases used were all standard grade analyte mixtures in a balance of nitrogen. Six gas analytes (acetaldehyde (5.08 ppm), acetone (5.50 ppm), benzene (5.10 ppm), ethanol (5.00 ppm and 9.48 ppm), formaldehyde (5.05 ppm), and methanol (4.66 ppm)) were evaluated, all from Praxair, California, USA.

Initially, a "blank" (empty round bottom flask) was run to measure the initial concentration of the gas analyte. Before each polymeric material was tested, it was purged with 5.0 grade nitrogen (Praxair, Mississauga, Canada) for 60 minutes. Then the round bottom flask was connected to the test system and exposed to the gas analytes for 72 minutes where the GC measured the amount of analyte not sorbed (residual analyte) onto the polymeric material. A measurement was taken at 60 minutes and 72 minutes to ensure equilibrium had been reached. The amount of gas analyte was calculated by subtracting the residual analyte measured by the GC from the initial concentration of the gas analyte (empty flask).

One gas mixture was also tested. To mix the gases, two MFCs were used (one for each gas analyte) and the gases were mixed in a passive mixture inline. The flow rates were varied such that a concentration of $\sim 4 \mathrm{ppm}$ of acetaldehyde and $\sim 6 \mathrm{ppm}$ of ethanol was used. Given the concentrations available, this was as close to $5 \mathrm{ppm}$ for each analyte that could be achieved in the gas mixture. 


\section{Results and Discussion}

\subsection{Characterization}

The polymeric materials were characterized using energy dispersive $\mathrm{x}$-ray (EDX) to measure the amount of metal oxide incorporated into the P25DMA (see Table 1) and scanning electron microscopy (SEM) to observe the morphology (Figure 2). Note that the distribution of the metal oxide nanoparticles cannot be seen in the SEM images since the metal oxide nanoparticles reside within the polymer matrix. The "bright spots" (such as in Figure 2c) on the SEM images are actually sulfur clusters $\left(\mathrm{SO}_{4}{ }^{2-}\right.$ from the ammonium persulfate initiator) that have bound to the polymer. However, multiple EDX measurements on replicate nanocomposite samples exhibited similar amounts of the metal oxide. Therefore, it can be concluded that the metal oxide nanoparticle distribution was homogenous for all practical purposes.

Table 1. Amount of Metal Oxide Nanoparticles Incorporated into the P25DMA

\begin{tabular}{ll}
\hline Metal & Weight Percent \\
\hline $\mathrm{Al}$ & $0.61 \%$ \\
$\mathrm{Ni}$ & $5.58 \%$ \\
$\mathrm{Ti}$ & $3.68 \%$ \\
$\mathrm{Zn}$ & $0.34 \%$ \\
\hline
\end{tabular}



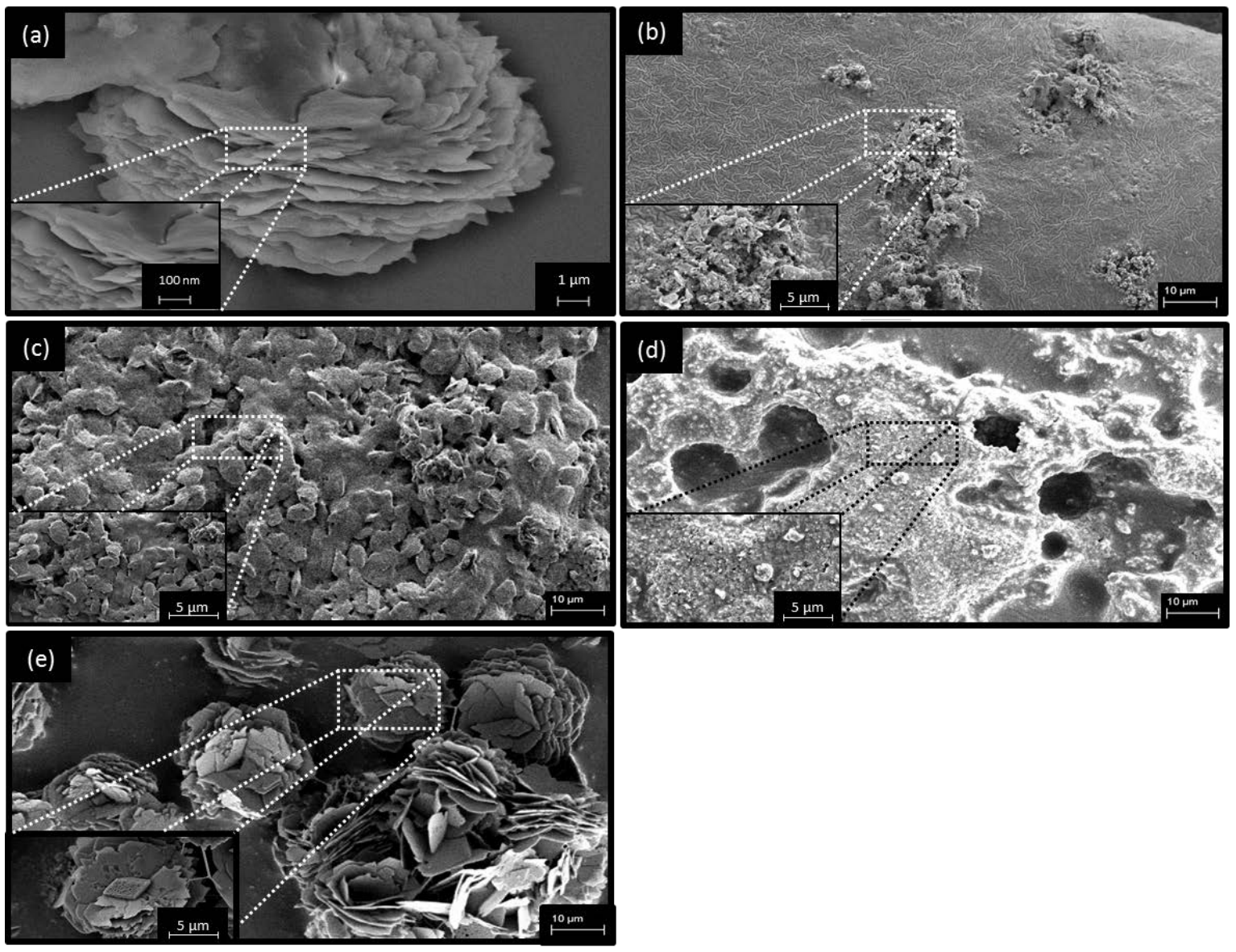

Figure 2. SEM images of (a) P25DMA, (b) P25DMA 5\% $\mathrm{Al}_{2} \mathrm{O}_{3}$, (c) P25DMA 5\% NiO, (d) P25DMA 5\% $\mathrm{TiO}_{2}$, and (e) P25DMA 5\% ZnO.

Note that for each of the doped P25DMA materials, $5 \mathrm{wt}$. \% of the metal oxide was available during synthesis. If all of the metal oxide were incorporated, then EDX would in principle show 5 wt. \% incorporated into each polymeric material. This, however, was not the case for $\mathrm{Al}$ and $\mathrm{Zn}$. Despite only a small amount of the metal oxide nanoparticles binding to the P25DMA, the morphology of the polymer matrix (see Figure 2b-e) was altered from the morphology of the undoped P25DMA (see Figure 2a). In general, as the amount of metal oxide nanoparticles incorporated increased, the morphology deviated more from the morphology of the undoped P25DMA. For example, the morphology of the P25DMA 5\% $\mathrm{ZnO}$ (see Figure 2e), which only incorporated $0.34 \% \mathrm{ZnO}$, was very similar to that of the undoped P25DMA (see Figure 2a), whereas the morphology of the P25DMA 5\% NiO (see Figure 2c), which incorporated $5.58 \%$ had a significantly different morphology. The undoped P25DMA and the P25DMA 5\% ZnO had many thin layers stacked on top of one another similar to a flower and the P25DMA5\% NiO was very globular.

The type of metal oxide present during synthesis affected the resulting P25DMA polymer 
nanocomposite. Not all of the metal oxides were incorporated well into the P25DMA matrix. In addition, the morphology of the P25DMA nanocomposites varied when different metal oxides were present (Figure 2). Therefore, despite not all of the metal oxide incorporating into the P25DMA matrix, the metal oxide nanoparticles still influenced the morphology of the resulting polymer nanocomposite.

\subsection{Sorption tests}

Each of the five sensing materials was evaluated for selectivity, individually. Each sensing material was exposed to approximately 5 ppm of each of the six gas analytes (acetaldehyde, acetone, benzene, ethanol, formaldehyde, and methanol). The amount of gas analyte that sorbed onto each sensing material is shown in Figure 3.

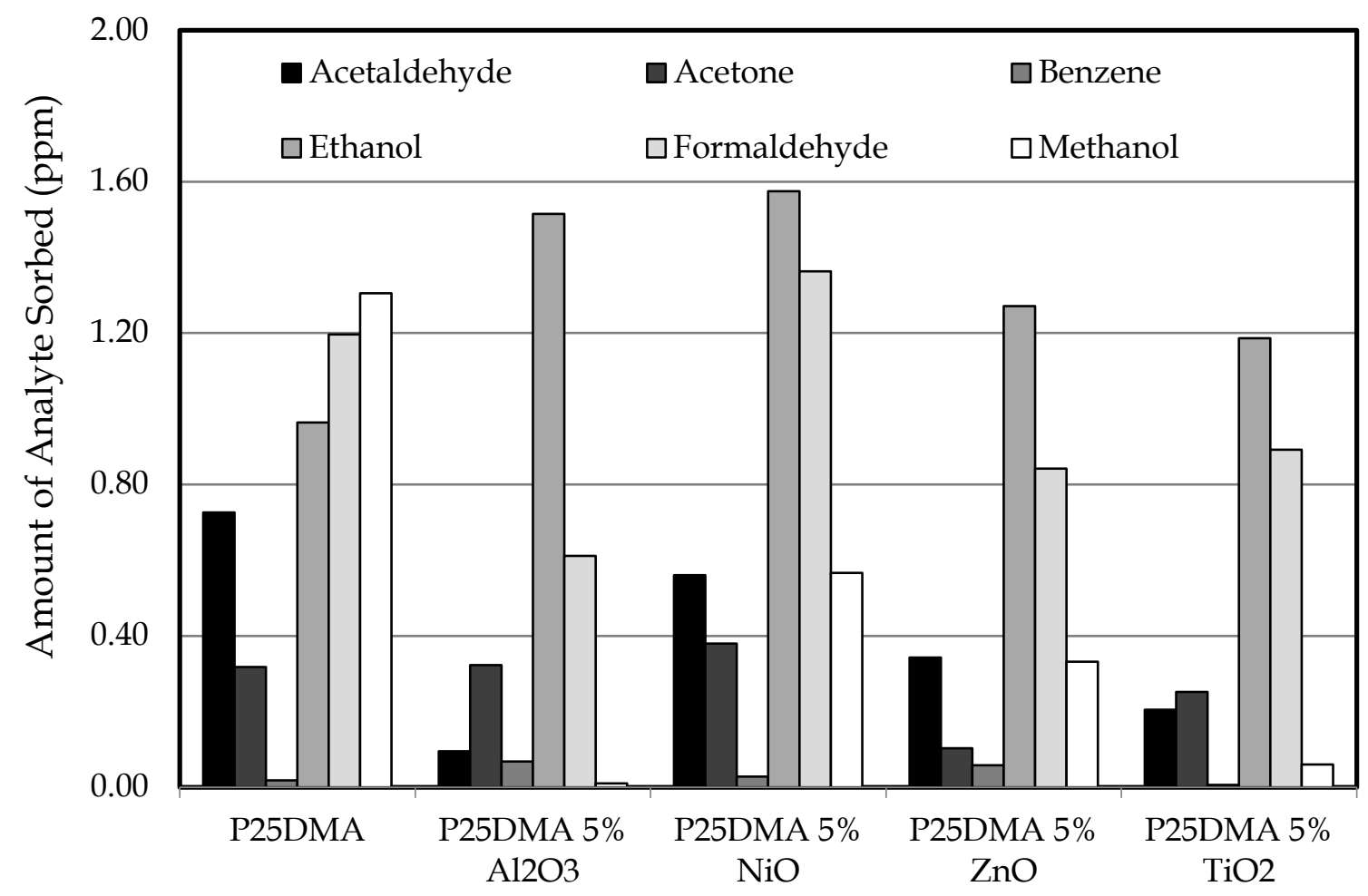

Figure 3. Amount of gas analyte sorbed onto each sensing material. Note that from left to right, the bars represent acetaldehyde, acetone, benzene, ethanol, formaldehyde, and methanol, respectively.

From Figure 3, it can be seen that none of the sensing materials are particularly selective to any of the gas analytes evaluated. Although, it should be noted that all of these sensing materials are very sensitive to six gas analytes evaluated since gas sorption (i.e. a response) was observed when these sensing materials were exposed to $5 \mathrm{ppm}$ of gas analyte, which is 
a very low concentration to detect.

The most selective sensing material was P25DMA 5\% $\mathrm{Al}_{2} \mathrm{O}_{3}$, which is fairly selective towards ethanol. For comparison, P25DMA had the worst selectivity towards any of the gas analytes. Therefore, as a single sensing material on a gas sensor, none of these sensing materials, with the possible exception of P25DMA 5\% $\mathrm{Al}_{2} \mathrm{O}_{3}$, would have the required selectivity. However, the partial selectivity could be exploited on a sensor array.

\subsection{Sensor Array}

To improve the selectivity of a sensor, multiple sensing materials can be used in an array. By combining the responses on multiple sensing materials, different gas analytes can be separated using a statistical algorithm. These algorithms compare the different responses from different analytes makes on different sensing materials.

A common algorithm used is principle component analysis (PCA). PCA is a multivariate statistical method that converts an array of data into principal components that are a linear combination of the original variables. The goal is to reduce the number of principal components, where the maximum number of principal components is equal to the total number of variables, while retaining the maximum amount of variability. Therefore, only the first few principal components are typically used, where the first principal component contains the most variance and the $\mathrm{n}^{\text {th }}$ principal component contains the least. ${ }^{[19]}$

As an algorithm for a sensor array, only the first two principal components are typically used (Factor 1 and Factor 2) since these two principals contain the most variance. In this case, the variables were the sensing materials and the data were grouped by gas analyte. This forced the data (amounts sorbed) to correlated by gas analyte, resulting in gas analytes being separated into clusters when Factor 1 was plotted against Factor 2. Note that this analysis does not provide any information on how correlated specific variables are.

The data collected from the sorption tests in the previous section were entered into the algorithm. Four replicates for each sensing material-gas analyte combination was used in the PCA. The resulting Factor 1 and Factor 2 (the first two principal components) were plotted for each point (Figure 4). This graph is the reference or calibration graph with which 
unknown gases are compared to.

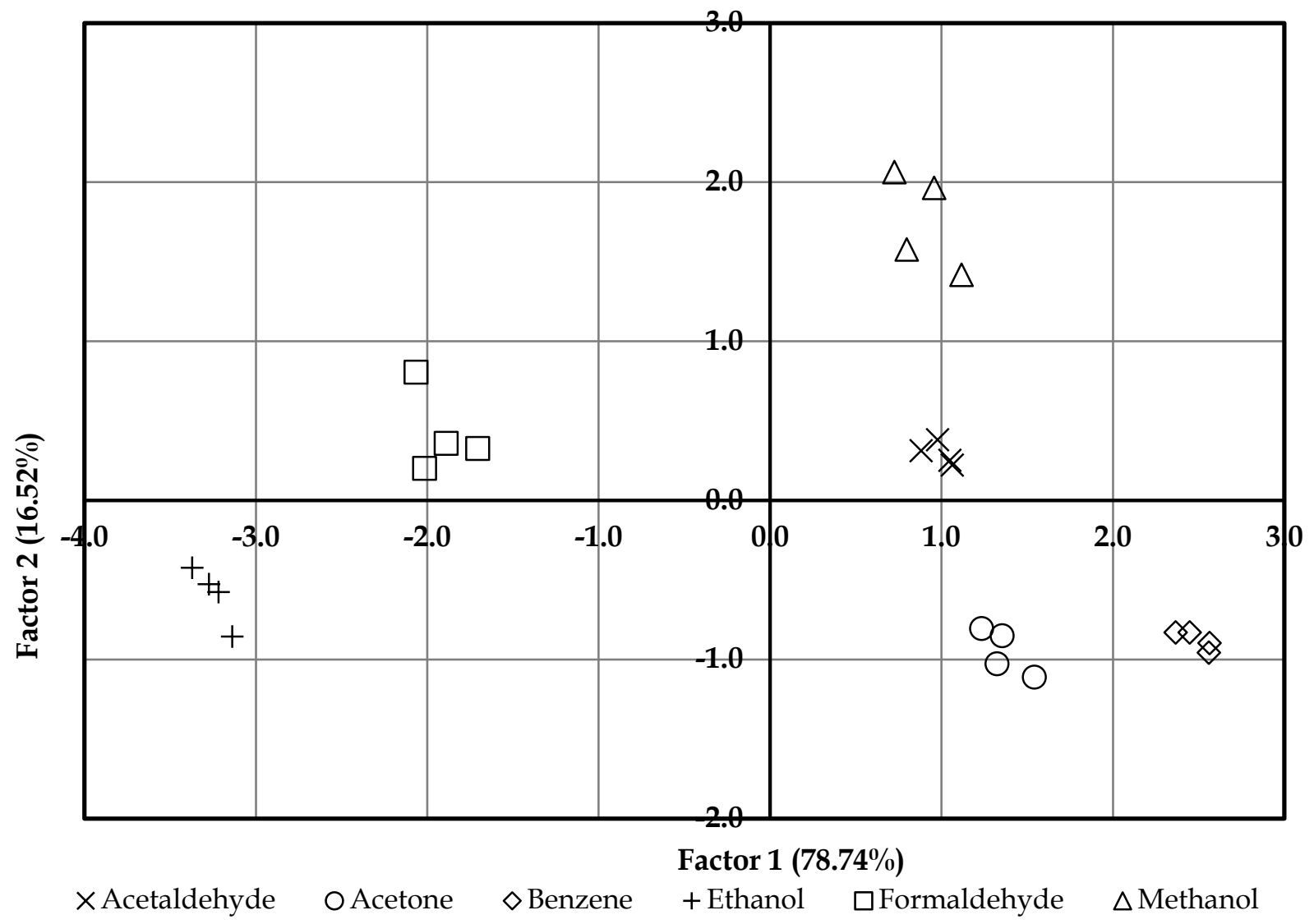

Figure 4. PCA reference plot.

Using the plot in Figure 4 as a reference, six unknown gases were measured. Each of these gases was singular in nature (i.e. only one gas analyte was measured at a time). The resulting points for each unknown, after being analyzed using PCA, were plotted on top of the reference graph (Figure 5). In all cases, the unknown could be easily identified since the response on the unknown landed very close to the previously identified gas clusters. This is confirmation that the reference plot (Figure 4) could be used to identify these six (single) gases and therefore, acts as proof-of-concept for individual gas analytes. 


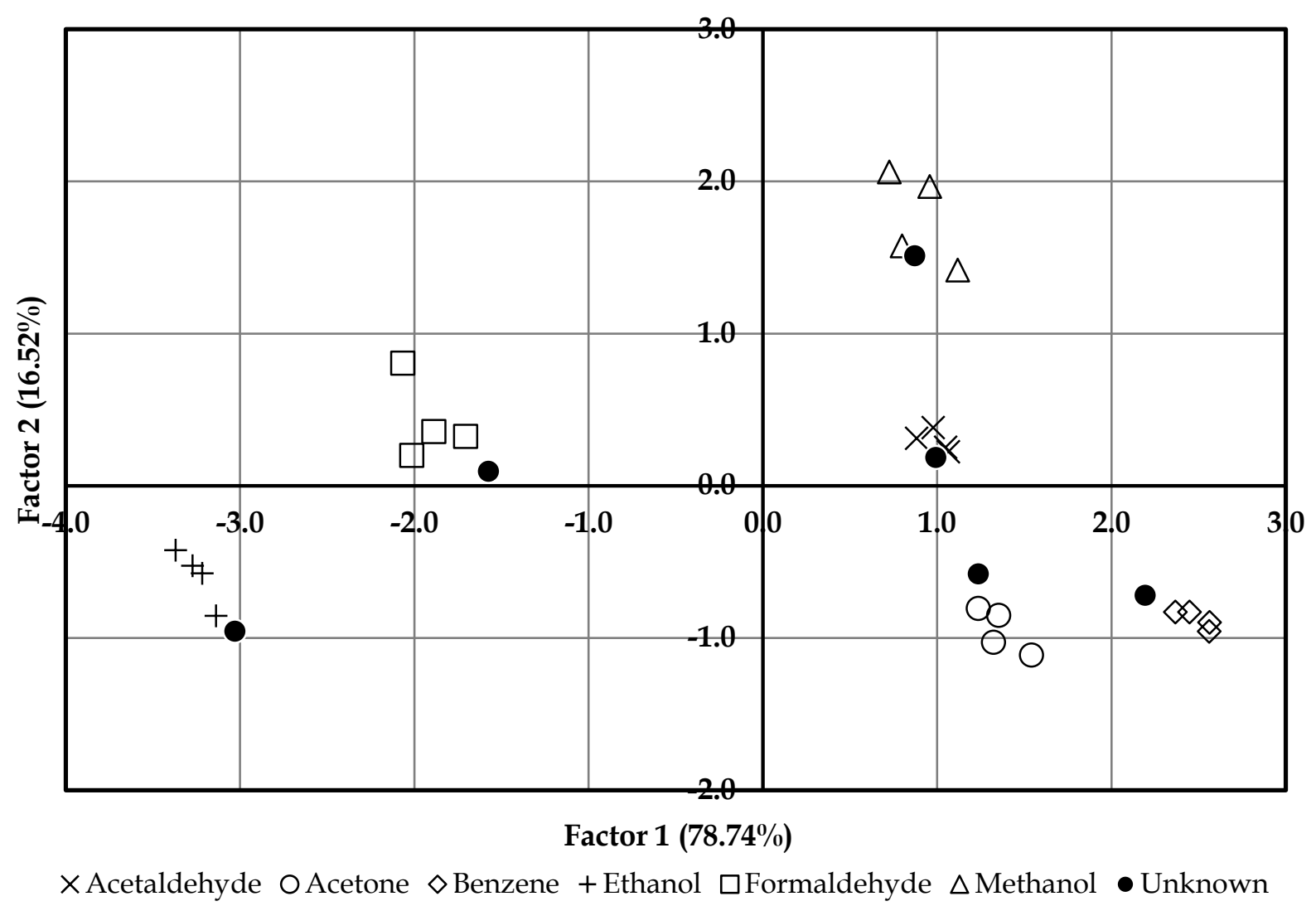

Figure 5. PCA plot with unknowns

A gas mixture was also evaluated (again for initial proof-of-concept), using ethanol and acetaldehyde in a 2:1 ratio. Two replicates were run, which produced four points (two for ethanol and two for acetaldehyde). These points landed partway between ethanol and acetaldehyde on the reference plot (Figure 6). In addition, these points did not overlap with any of the other gas clusters. 


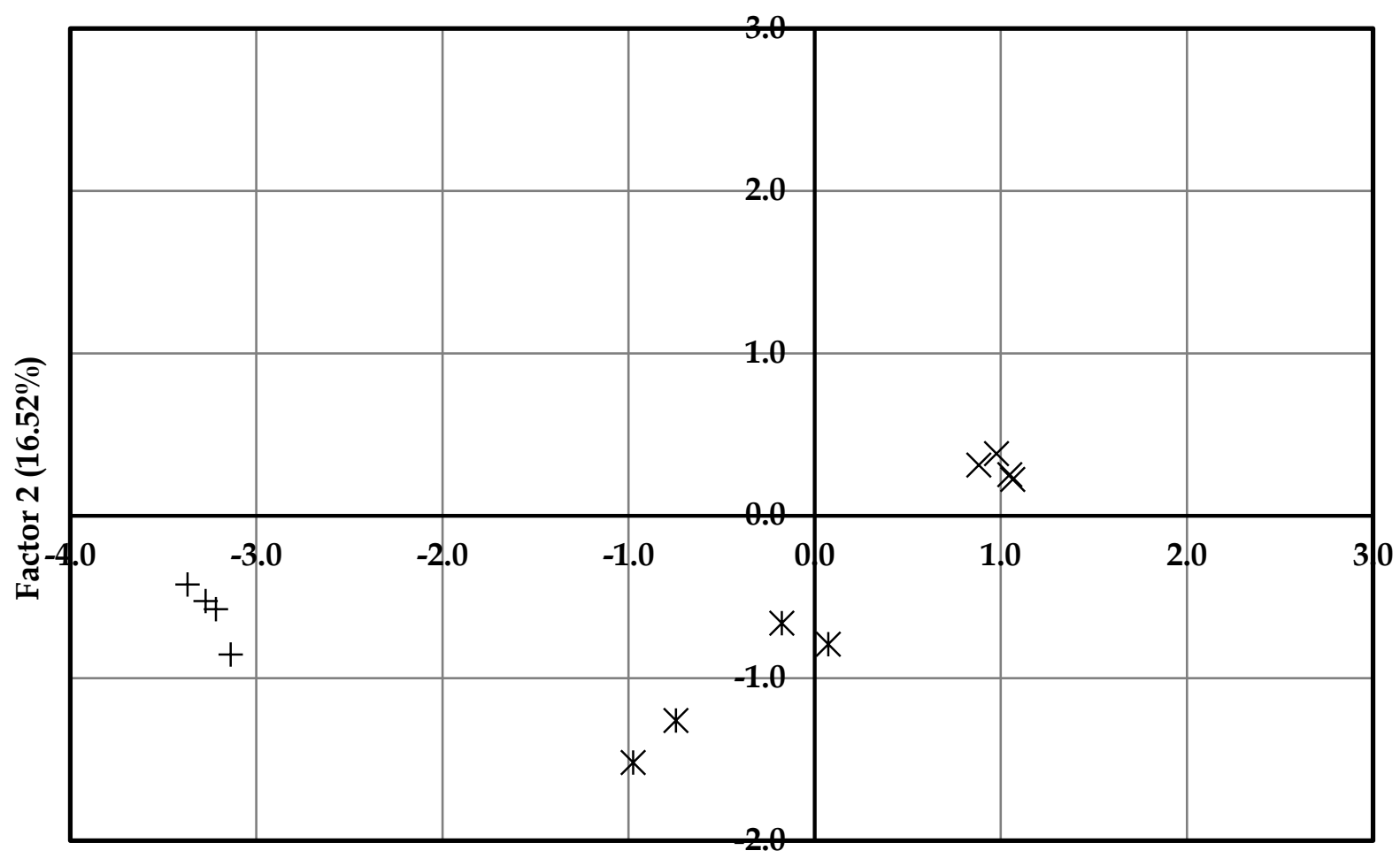

Factor $1(78.74 \%)$

$\times$ Acetaldehyde + Ethanol $*$ Gas Mixture

Figure 6. Gas mixture

It should be noted that an interaction between acetaldehyde and ethanol did occur (i.e. the presence of acetaldehyde did affect how much ethanol sorbed and vice versa). In this case, ethanol facilitated the sorption of acetaldehyde resulting in acetaldehyde more readily sorbing than ethanol. Since the sorption of the analytes was affected by more than one being present in the mixture, a separate cluster for the binary mixture of ethanol and acetaldehyde (as seen in Figure 6) was within reason.

Given that gas analytes interact when present with one another, these interactions in binary, ternary, etc. gas mixtures should be further evaluated to improve the algorithm and thus better separate gas analytes in these mixtures. However, for single gas analytes, a sensor array consisting of these five sensing materials (P25DMA, P25DMA 5\% $\mathrm{Al}_{2} \mathrm{O}_{3}$, P25DMA 5\% NiO, P25DMA 5\% $\mathrm{TiO}_{2}$, and P25DMA 5\% $\mathrm{ZnO}$ ) is able to distinguish between six different gas analytes (acetaldehyde, acetone, benzene, ethanol, formaldehyde, and methanol).

Note that this is a first attempt (proof-of-concept) and seems quite promising. Of course, 
the more data points PCA uses, the better the data correlation structure will be identified. With a more comprehensive data set and filtering algorithm, these sensing materials could be deposited as thin films onto some type of sensor array, such as a mass-based sensor ${ }^{[20]}$ or a capacitive-based sensor ${ }^{[21]}$.

\section{Conclusions}

Five sensing materials (P25DMA, P25DMA 5\% $\mathrm{Al}_{2} \mathrm{O}_{3}$, P25DMA 5\% NiO, P25DMA 5\% $\mathrm{TiO}_{2}$, and P25DMA 5\% ZnO) were evaluated as potential sensing materials for a gas sensor array to monitor indoor air quality. Each sensing material was evaluated individually using six different VOCs (acetaldehyde, acetone, benzene, ethanol, formaldehyde, and methanol). It was found that individually, none of these sensing materials were very selective towards any gas analyte, especially formaldehyde and benzene. It should be noted that P25DMA $5 \% \mathrm{Al}_{2} \mathrm{O}_{3}$ was selective towards ethanol.

By combining the five sensing materials into a sensor array, and using PCA as the filtering algorithm, six different gas analytes (acetaldehyde, acetone, benzene, ethanol, formaldehyde, and methanol) could be identified. A reference plot was created with which six different unknown gas samples containing a single gas analyte were compared. In all six cases, the unknown was correctly identified.

A gas mixture using acetaldehyde and ethanol was also evaluated; however, gas mixture could not be separated. This was due to only single gas analytes being used to create the reference plot with which the gas mixture was compared to, although the mixture did fall on the reference plot between the two gas analytes (acetaldehyde and ethanol), which was expected. To improve this sensor array, a more sophisticated filtering algorithm is needed; however, for single gas analytes, this sensor array is capable of detecting low concentrations (5 ppm) of six different gas analytes (VOCs), including formaldehyde and benzene.

\section{References}

[1] S. K. Brown, Indoor Air 2002, 12, 55.

[2] J. González-Chavarri, I. Castro-Hurtado, I. Ayerdi, E. Castaño, G. G. Mandayo, IEEE CDE Madrid, Spain, February 11-13, 2015.

[3] B. Li, J. Liu, G. Shi, J. Liu, Sensor Actuat B 2013, 177, 1167.

[4] M. De Wit, E. Vanneste, H. J. Geise, L. J. Nagels, Sensor Actuat B 1998, 50, 164.

[5] N. H. Beltrán, M. A. Duarte-Mermoud, V. A. Soto, S. A. Salah, M. A. Bustos, Int J Elec Comp Eng 2006, 1, 378 . 
[6] V. Talwar, O. Singh, R. C. Singh, Sensor Actuat B 2014, 191, 276.

[7] S. Rochat, T. M. Swager, ACS Appl Mater Int 2013, 5, 4488.

[8] M. F. Mabrook, C. Pearson, M. C. Petty Sensor Actuat B 2006, 115, 547.

[9] K. M. E. Stewart, W. T. Chen, R. R. Mansour, A. Penlidis, J Appl Polym Sci 2015, 132, 42259.

[10] D. R. Patil, L. A. Patil, D. P. Amalnerkar, B Mater Sci 2007, 30, 553.

[11] M. K. Deore, G. H. Jain, J Petrol Sci Res 2014, 3, 60.

[12] Z. Lou, L. Wang, T. Fei, T. Zhang, New J Chem 2012, 36, 1003.

[13] K. M. E. Stewart, N. T. McManus, E. Abdel-Rahman, A. Penlidis, J Macromolec Sci A 2012, 49, 1.

[14] J. Zheng, G. Li, X. Ma, Y. Wang, G. Wu, Y. Cheng, Sensor Actuat B 2008, 133, 374.

[15] K. I. Arshak, L. M. Cavanagh, I. Gaidan, E. G. Moore, S. A. Clifford, R. Phelan, C. Cunniffe, J. A. Harris, G. M. Lyons, IEEE Sens 2004, 681.

[16] B. L. Zhu, C. S. Xie, W. Y. Wang, K. J. Huang, J. H. Hu, Mater Lett 2004, 58, 624.

[17] R. Zou, G. He, K. Xu, Q. liu, Z. Zhang, J. Hu, J Mater Chem A 2013, 1, 8445.

[18] K. M. E. Stewart, A. Penlidis, Macromol Sy 2013, 324, 11.

[19] A. J. Scott, A. Penlidis, J Macromol Sci A 2013, 50, 803.

[20] M. K. Khater, M. Al-Ghamdi, S. Park, K. M. E. Stewart, E. M. Abdel-Rahman, A. Penlidis, A. H. Nayfeh, A. K. S Abdel-Aziz, M. Basha, J Micromech Microeng 2014, 24, 065007.

[21] W.-T. S. Chen, K. M. E. Stewart, C. K. Yang, R. R. Mansour, J. Carroll, A. Penlidis, IEEE Trans Microw Theory Tech 2015, 60, 4157. 